\title{
Sum-Frequency Vibrational Spectroscopy on Chiral Liquids: A Novel Technique to Probe Molecular Chirality
}

\author{
M. A. Belkin, T. A. Kulakov, K.-H. Ernst, ${ }^{*}$ L. Yan, ${ }^{\dagger}$ and Y. R. Shen ${ }^{\ddagger}$ \\ Department of Physics, University of California, Berkeley, California 94720 \\ and Materials Sciences Division, Lawrence Berkeley National Laboratory, Berkeley, California 94720
}

(Received 25 May 2000)

\begin{abstract}
Optical activity in sum-frequency vibrational spectra has been observed for the first time in chiral liquids. The electric-dipole allowed chiral element of the nonlinear susceptibility appears to be 3 orders of magnitude smaller than typical allowed achiral elements. This is partly because the observed chirality requires a breakdown of the Born-Oppenheimer approximation.
\end{abstract}

PACS numbers: 33.55.Ad, 33.20.Tp, 42.65.-k, 78.20.Ek

Chiral molecules play an important role in molecular science including biology and medicine [1]. Optical circular birefringence $(\mathrm{CB})$, circular dichroism $(\mathrm{CD})$, and Raman optical activity (ROA) are the usual techniques used to probe molecular chirality [2]. Recently, nonlinear optical studies of chiral molecules have attracted a great deal of attention. Hicks and co-workers first demonstrated the existence of optical activity in second-harmonic generation (SHG) from a surface monolayer of chiral molecules [3]. Persoons and co-workers also studied SHG from chiral surfaces, monolayers, and films [4]. The main interest in SHG as a chirality probe lies in the fact that the optical coefficients for $\mathrm{CB}, \mathrm{CD}$, and ROA are electric-dipole forbidden, while the surface nonlinearity for optically active SHG is electric-dipole allowed.

It seems natural to extend SHG studies of chirality to sum-frequency generation (SFG). With tunable infrared sources, SFG can be used as a vibrational spectroscopic tool [5] and therefore can be a much more powerful probe than SHG. It would allow probing of chirality in vibrational modes associated with selected atomic groups within a chiral molecule.

Like SHG, SFG is electric-dipole allowed at a liquid surface, but, unlike SHG, the chiral components of SFG are also electric-dipole allowed by symmetry in a liquid bulk [6]. Then, as Giordmaine suggested in 1965 [6], the optically active SFG from the bulk of a chiral liquid would be easily observable. Rentzepis $e t$ al. did report the observation of the effect in 1966 [7], but their results have not yet been definitely confirmed [8].

In this paper we report the first measurement of optically active SFG vibrational spectra from a number of chiral liquids. We demonstrate the applicability of SFG as a vibrational spectroscopic tool to study chirality, and also confirm experimentally the existence of electric-dipole allowed SFG from the bulk of chiral liquids. We have found that, although the optically active SFG signal enhanced by vibrational resonances is readily detectable, its strength is rather weak, corresponding to a nonlinearity 3 orders smaller than the typical electric-dipole allowed nonlinearity. We understand that this is because the BornOppenheimer (adiabatic) approximation is not strictly valid and the chirality in an inherently achiral atomic group, such as $\mathrm{CH}_{2}$ or $\mathrm{CH}$, is present only because of perturbation from the chiral molecular structure. In our case, the chiral SFG signal is observable in transmission owing to the relatively long coherence length of the interaction, but it is too weak to be observed in reflection or from a surface monolayer.

Consider two input waves $\vec{E}_{1}$ and $\vec{E}_{2}$ at frequencies $\omega_{1}$ (visible) and $\omega_{2}$ (infrared) with wave vectors $\vec{k}_{1}$ and $\vec{k}_{2}$, respectively, interacting in a medium. The effective nonlinear polarization induced can be written in the form [9]

$$
\begin{aligned}
\vec{P}_{\mathrm{eff}}^{(2)}(\omega)= & \vec{P}^{(2)}(\omega)-\vec{\nabla} \cdot \vec{Q}^{(2)}(\omega)+\frac{i}{\omega} \vec{\nabla} \times \vec{M}^{(2)}(\omega)+\ldots, \\
\vec{P}^{(2)}(\omega)= & \epsilon_{0} \overleftrightarrow{\chi}^{e e e}: \vec{E}_{1} \vec{E}_{2}+\epsilon_{0} \vec{\chi}^{e q e}: \vec{k}_{1} \vec{E}_{1} \vec{E}_{2}+\epsilon_{0} \vec{\chi}^{e e q}: \vec{k}_{2} \vec{E}_{1} \vec{E}_{2}+\epsilon_{0} \overleftrightarrow{\chi}^{e m e}:\left(\vec{k}_{1} \times \vec{E}_{1}\right) \vec{E}_{2} \\
& +\epsilon_{0} \vec{\chi}^{e e m}: \vec{E}_{1}\left(\vec{k}_{2} \times \vec{E}_{2}\right) \\
\vec{Q}^{(2)}(\omega)= & \epsilon_{0} \overleftrightarrow{\chi}^{q e e}: \vec{E}_{1} \vec{E}_{2} \\
\vec{M}^{(2)}(\omega)= & \omega \epsilon_{0} \stackrel{\leftrightarrow}{\chi}^{m e e}: \vec{E}_{1} \vec{E}_{2}
\end{aligned}
$$

where $\omega=\omega_{1}+\omega_{2}, \vec{P}^{(2)}, \vec{Q}^{(2)}$, and $\vec{M}^{(2)}$ denote the electric-dipole polarization, electric-quadrupole polarization, and magnetization, respectively, and $\overleftrightarrow{\chi}^{\prime}$ 's are the secondorder nonlinear susceptibilities.
In an achiral liquid, symmetry arguments yield $\vec{\chi}^{e e e}=$ 0 , and the only nonvanishing elements stem from electricquadrupole and magnetic-dipole allowed $\vec{\chi}_{Q M}$ (we shall use $\vec{\chi}_{Q M}$ to represent $\vec{\chi}^{e q e}, \overleftrightarrow{\chi}^{\text {eme }}$, etc.) [10]. In a chiral 
liquid, however, $\chi_{i j k}^{e e e}=\chi_{\text {chiral }} \cdot e_{i j k}$, where $e_{i j k}$ is the Levi-Civita symbol, does not vanish. There exist no chiral elements (i.e., the elements that are different for different enantiomers) in $\overleftrightarrow{\chi}_{Q M}$. If $\chi_{\text {chiral }}$ were of the same order of magnitude as a typical electric-dipole allowed element of $\vec{\chi}^{(2)}$, e.g., that of quartz, we would expect to observe optically active SFG easily.

In terms of the nonlinear polarizability elements $\alpha_{\xi \eta \zeta}^{(2)}$ of a molecule, we have [6]

$$
\chi_{\text {chiral }}=\frac{1}{\epsilon_{0}} N L(\omega) L\left(\omega_{1}\right) L\left(\omega_{2}\right) \cdot \alpha_{\text {chiral }},
$$

where

$$
\begin{aligned}
& \alpha_{\text {chiral }} \\
& \equiv \frac{\alpha_{\xi \eta \zeta}^{(2)}-\alpha_{\xi \zeta \eta}^{(2)}+\alpha_{\zeta \xi \eta}^{(2)}-\alpha_{\zeta \eta \xi}^{(2)}+\alpha_{\eta \zeta \xi}^{(2)}-\alpha_{\eta \xi \zeta}^{(2)}}{6} .
\end{aligned}
$$

Here $\xi, \eta$, and $\zeta$ are three different molecular coordinates, $N$ is the number of molecules per unit volume, and $L\left(\omega_{i}\right)=\frac{\epsilon\left(\omega_{i}\right)+2}{3}$ is the Lorentz local-field correction factor at $\omega_{i}$ with $\epsilon\left(\omega_{i}\right)$ being the dielectric constant at $\omega_{i}$. If $\omega_{2}$ is near a vibrational resonance, then the resonant part of $\vec{\alpha}^{(2)}$ can be expressed as [11]

$$
\left(\alpha_{q}^{(2)}\right)_{\xi \eta \zeta}=\frac{1}{2 \omega_{q}} \frac{\partial \alpha_{\xi \eta}}{\partial Q_{q}} \frac{\partial \mu_{\zeta}}{\partial Q_{q}} \frac{1}{\omega_{2}-\omega_{q}+i \Gamma_{q}},
$$

where $\omega_{q}$ is the resonance frequency, $Q_{q}$ is the normal coordinate, $\frac{\partial \alpha_{\xi_{\eta}}}{\partial Q_{q}}$ is the Raman polarizability element, and $\mu_{\zeta}$ is the dipole moment along $\zeta$ for the $q$ th vibrational mode. It was shown in Ref. [12] that, for $\omega_{1}$ and $\omega$ far from resonance (as in our experiment), the antisymmetric Raman components are appreciable only when the adiabatic approximation for electron-vibrational coupling breaks down, but still only $\sim 10^{-2}$ times as large as the symmetric components of $\frac{\partial \alpha_{\xi \eta}}{\partial Q_{q}}$. We therefore expect $\alpha_{\text {chiral }}$ to be no more than $\sim 10^{-2}$ times the typical achiral element of $\vec{\alpha}^{(2)}$, or electric-dipole allowed $\chi_{\text {chiral }}$ to be about the same as the elements of $\vec{\chi}_{Q M}$.

The SFG signal with the $\left(\hat{e}, \hat{e}_{1}, \hat{e}_{2}\right)$ input/output polarization combinations is generally given by [5]

$$
\begin{aligned}
I(\omega) & =\frac{\omega^{2}}{8 \epsilon_{0} c^{3} \cos ^{2}(\beta)}\left|\chi_{\mathrm{eff}}^{(2)}\right|^{2} I_{1}\left(\omega_{1}\right) I_{2}\left(\omega_{2}\right), \\
\chi_{\mathrm{eff}}^{(2)} & =[\vec{F}(\omega) \cdot \hat{e}] \cdot \stackrel{\leftrightarrow}{\chi}_{T}^{(2)}:\left[\vec{F}\left(\omega_{1}\right) \cdot \hat{e}_{1}\right]\left[\vec{F}\left(\omega_{2}\right) \cdot \hat{e}_{2}\right], \\
\vec{\chi}_{T}^{(2)} & =\vec{\chi}_{S}^{(2)}+\overleftrightarrow{\chi}_{B}^{(2)} / \Delta k_{z}, \\
\Delta k_{z} & =k_{z}-k_{1 z}-k_{2 z},
\end{aligned}
$$

where $\omega=\omega_{1}+\omega_{2}$ and $\vec{k}$ are the frequency and the wave vector of sum-frequency (SF) beam, $I_{i}\left(\omega_{i}\right)$ is the beam intensity at $\omega_{i}, \beta$ is the exit angle of the SF beam in air, $\vec{F}\left(\omega_{i}\right)$ is the tensorial Fresnel transmission factor at $\omega_{i}$ at the interface, $\dddot{\chi}_{S}^{(2)}$ is the surface nonlinear susceptibility, $\leftrightarrow_{\mathcal{X}}^{(2)} \equiv \vec{P}_{\text {eff }}^{(2)} /\left(\vec{E}_{1} \vec{E}_{2}\right)$ is the bulk nonlinear susceptibility $\left\{\left[\chi_{B}^{(2)}\right]_{i j k}(i \neq j \neq k)=\chi_{\text {chiral }} \cdot e_{i j k}\right.$ is responsible for the optically active SFG $\}, l_{\text {coh }} \equiv 1 /\left|\Delta k_{z}\right|$ is the coherence length of the interaction, and $\hat{z}$ is along the surface normal. Equation (4) is valid for SFG in both transmission and reflection, but $l_{\text {coh }}$ is about 20 times larger for transmission than for reflection. Therefore the contribution from $\vec{\chi}_{B}^{(2)}$ to the SFG signal is much weaker in reflection $[\sim 400$ times weaker than in transmission if the contribution from $\overrightarrow{\mathcal{X}}_{S}^{(2)}$ is negligible]. Both $\overleftrightarrow{\mathcal{X}}_{S}^{(2)}$ and $\overleftrightarrow{\mathcal{X}}_{B}^{(2)}$ can be decomposed into a resonant part and a nonresonant background. We can write, in general,

$$
\begin{aligned}
& \overleftrightarrow{\chi}_{S}^{(2)}=\overleftrightarrow{\chi}_{S}^{N R}+\overleftrightarrow{\chi}_{S}^{R}, \\
& \overleftrightarrow{\mathcal{\chi}}_{B}^{(2)}=\overleftrightarrow{\mathcal{\chi}}_{B}^{N R}+\overleftrightarrow{\chi}_{B}^{R},
\end{aligned}
$$

with $\vec{\chi}_{S, B}^{R}=\sum_{q} \frac{\left.\overleftrightarrow{(A}_{q}\right)_{S, B}}{\omega_{1}-\omega_{q}+i \Gamma_{q}}$. With this expression for $\overleftrightarrow{\chi}_{S}^{(2)}$ and $\overleftrightarrow{\chi}_{B}^{(2)}$, Eq. (4) describes the SFG vibrational spectrum.

To access $\chi$ chiral of a chiral liquid by SFG, we must have components of $\vec{E}\left(\omega_{1}\right), \vec{E}\left(\omega_{2}\right)$, and $\vec{P}_{\text {eff }}^{(2)}(\omega)$ mutually perpendicular to each other. This is achieved by overlapping the two input beams at an angle at the entrance to the liquid and having an input/output polarization combination of $S P P\left(\hat{e}, \hat{e}_{1}\right.$, and $\hat{e}_{2}$ being $S, P$, and $P$ polarized, respectively), $P S P$, or $P P S$. In such a geometry, no achiral component of $\dot{\chi}_{T}^{(2)}$ contributes to the SFG output. The achiral elements can be accessed separately by the $S S P$, $S P S$, and $P P P$ polarization combinations.

Our experimental arrangement, shown schematically in Fig. 1(a), is similar to the one described in [10]. The two input beams, one fixed at $0.532 \mu \mathrm{m}$ and the other tunable in the infrared between 3.6 and $3.2 \mu \mathrm{m}$ (2800$3100 \mathrm{~cm}^{-1}$ ), were generated by a mode-locked Nd:YAG laser system. They both had a pulse width of $\sim 25 \mathrm{psec}$ and a repetition rate of $10 \mathrm{~Hz}$. Their pulse intensities were 800 and $200 \mu \mathrm{J}$ per pulse, respectively. The two beams overlapped at $90^{\circ}$ over an area of $2.5 \times 10^{-3} \mathrm{~cm}^{2}$ on the sample. The SF output was detected by a gated detector system. The directions of SF output in both transmission and reflection were determined by the requirement $k_{1 x}+$ $k_{2 x}=k_{x}$, with $\hat{x}$ being along the surface. Tuning the infrared input over vibrational resonances of the sample yields the SFG vibrational spectrum. The samples we studied were chiral liquids in an optical cell with fused quartz windows. The cell thickness of $0.5 \mathrm{~mm}$ was much larger than $l_{\text {coh }}$. We shall focus our discussion on limonene [Fig. 1(b)].
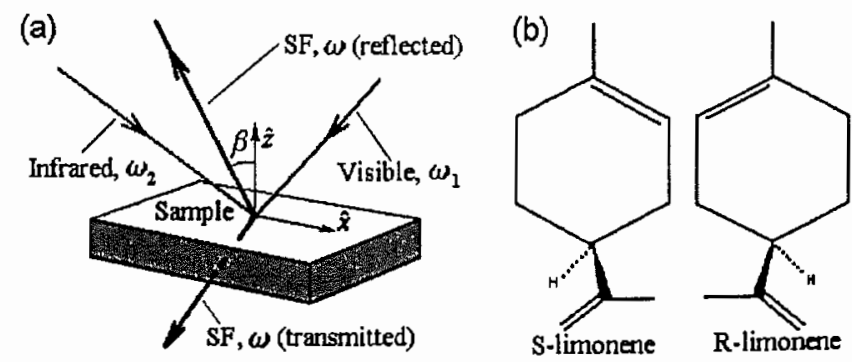

FIG. 1. (a) Experimental setup. (b) Molecular structure of $S$ and $R$-limonene. 
Figure 2 displays the SFG spectra in the $\mathrm{CH}$ stretch region for the two enantiomers of limonene and their racemic mixture obtained in transmission with $S P P, P S P, S S P$, $P P P$, and SPS polarization combinations. The spectra were normalized with respect to a reference crystalline quartz plate. The "achiral" $S S P, P P P$, and SPS spectra come only from the achiral elements of $\stackrel{\leftrightarrow}{\chi}_{T}^{(2)}$ in Eq. (4), whereas the "chiral" SPP and PSP spectra originate only from the chiral elements. They are clearly very different, indicating that the vibrational modes with strong chirality are not necessarily the dominant modes in achiral SFG spectra.

The spectra in Fig. 2 provide the following information. (i) The achiral SFG spectra for the two limonene enantiomers and the racemic mixture appear to be the same. The chiral SFG spectra for the two enantiomers are also identical, and, for the racemic mixture, no chiral SFG signal can be detected. This clearly indicates that we have observed optically active SFG and the associated vibrational spectrum for chiral limonene. (ii) The peaks in the chiral spectra are $\sim 25$ times stronger than the ones in the achiral SPS spectrum. As will be discussed later, the $S P S$ spectrum is dominated by bulk contribution from the electric-dipole forbidden $\vec{\chi}_{Q M}$. From the chiral and achiral spectra and Eq. (4), we estimated $\chi_{\text {chiral }}$ and the elements of $\stackrel{\leftrightarrow}{\chi}_{Q M}$ and found that they have comparable magnitudes, as was predicted. (iii) The chiral spectra yield $\left|\chi_{\text {chiral }}\left(\omega_{1}\right)\right|^{2}$, which is shown in Fig. 3. Fitting $\left|\chi_{\text {chiral }}\left(\omega_{1}\right)\right|^{2}$ by Eqs. (4) and (5) allows us to deduce the characteristic parameters for each vibrational mode. They are shown in Table I. We assigned the observed resonances in the chiral spectra as follows [13]: symmetric $\mathrm{CH}_{2}$ stretch at $2839 \mathrm{~cm}^{-1}$, symmetric $\mathrm{CH}_{3}$ stretch at
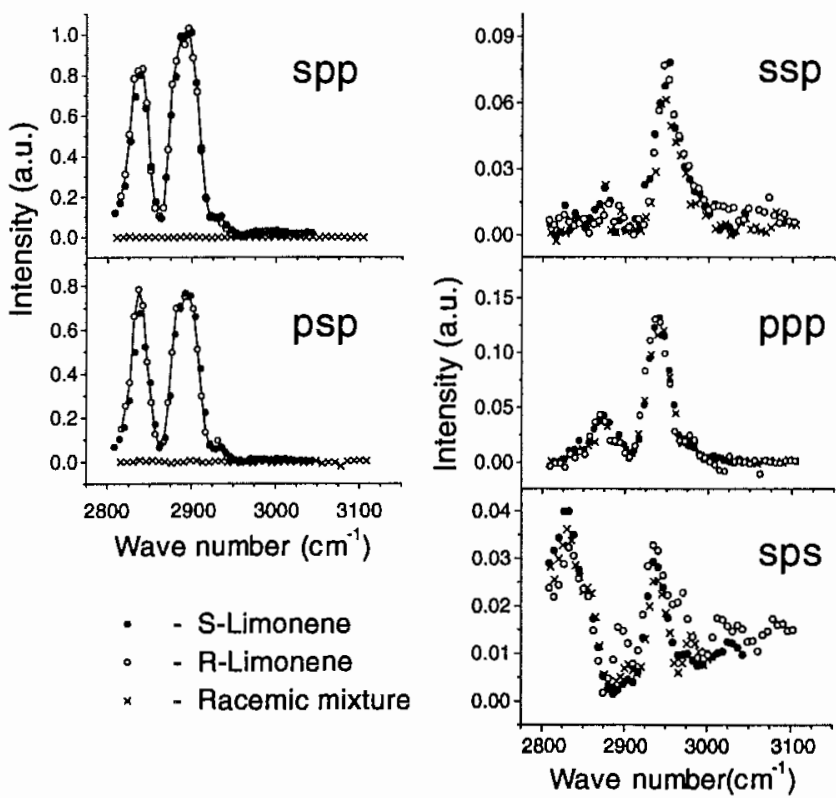

FIG. 2. SFG spectra of limonene samples in transmission in $S P P, P S P$ (chiral spectra), SSP, SPS, and PPP (achiral spectra) polarization combinations.
$2879 \mathrm{~cm}^{-1}, \mathrm{CH}$ stretch at $2905 \mathrm{~cm}^{-1}$, antisymmetric $\mathrm{CH}_{2}$ stretch at $2936 \mathrm{~cm}^{-1}$, and a very weak symmetric stretch of the $\mathrm{C}=\mathrm{C}$ double bonded $\mathrm{CH}_{2}$ group at $2990 \mathrm{~cm}^{-1}$ (not shown in the table). (iv) The values of $\left|\alpha_{\text {chiral }}\right|$ at resonance deduced from $\left|\chi_{\text {chiral }}\right|$ for different modes using Eq. (2) are also listed in Table I. They are more than 3 orders of magnitude smaller than the typical resonant value of $\sim 2 \times 10^{-50} \frac{\mathrm{m}^{3} \mathrm{C}}{\mathrm{V}^{2}}$ of an achiral element of $\stackrel{\leftrightarrow}{\alpha}^{(2)}$ for a $\mathrm{CH}_{x}$ stretch vibration [14]. The result supports our theoretical prediction discussed earlier.

As a chiral response, $\chi_{\text {chiral }}$ should change sign for the two enantiomers [6]. This can be checked by using $P M P$ input/output polarization combinations, where $M$ ("mixed") refers to a linear polarization of the visible beam at $\pm 45^{\circ}$ from its plane of incidence. From Eq. (4), we then have

$$
\left(\chi_{\mathrm{eff}}^{(2)}\right)_{ \pm}=\frac{1}{\sqrt{2}}\left[\left(\chi_{\mathrm{eff}}^{(2)}\right)_{P S P} \pm\left(\chi_{\mathrm{eff}}^{(2)}\right)_{P P P}\right]
$$

Therefore, the SFG output proportional to $\left|\left(\chi_{\text {eff }}^{(2)}\right)_{ \pm}\right|^{2}$ should display interference between the chiral and achiral contributions so that the spectra of $\left|\left(\chi_{\text {eff }}^{(2)}\right)_{+}\right|^{2}$ and $\left|\left(\chi_{\text {eff }}^{(2)}\right)-\right|^{2}$ are different.

Figure 4 shows that this is indeed the case for both enantiomers of limonene. With

$$
\begin{aligned}
\left|\left(\chi_{\text {eff }}^{(2)}\right)_{+}\right|^{2}-\left|\left(\chi_{\text {eff }}^{(2)}\right)_{-}\right|^{2}= & \left(\chi_{\text {eff }}^{(2)}\right)_{P S P}\left(\chi_{\text {eff }}^{(2)}\right)_{P P P}^{*} \\
& +\left(\chi_{\text {eff }}^{(2)}\right)_{P S P}^{*}\left(\chi_{\text {eff }}^{(2)}\right)_{P P P},
\end{aligned}
$$

we notice that, since $\left(\chi_{\mathrm{eff}}^{(2)}\right)_{P S P} \propto \chi_{\text {chiral }}$ changes sign when $R$-limonene is switched to $S$-limonene, the difference spectrum must be inverted, as actually seen in Fig. 4(c). Note that the peak strength in the difference spectra depends on the product of the chiral and achiral strengths at the same frequency.

We also measured SFG in reflection from the limonene liquids (limonene/quartz interface). No chiral signal could be distinguished from noise. The achiral SPS spectrum was also hardly observable. Since $l_{\text {coh }}$ is $\sim 20$ times less for SFG in reflection than in transmission, we can conclude, following Eq. (4), that the $S P S$ spectrum from transmission is dominated by the bulk contribution through $\stackrel{\leftrightarrow}{\chi}_{Q M}$.

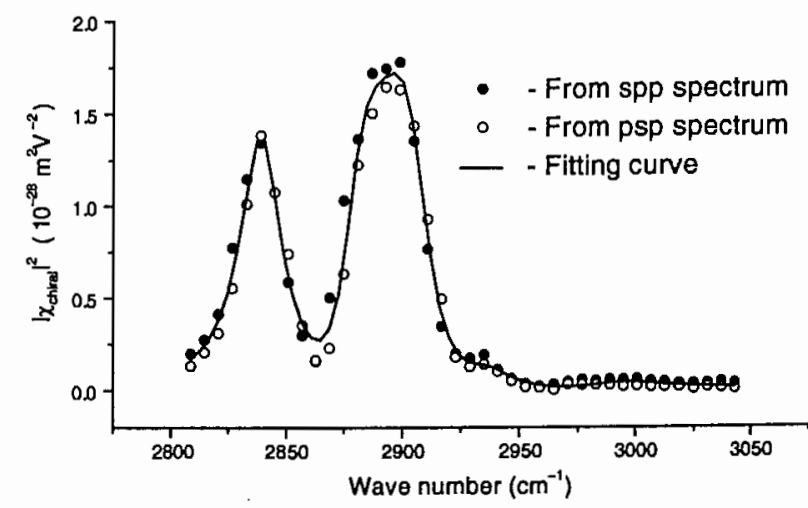

FIG. 3. Measured spectral dependence of $\left|\chi_{\text {chiral }}\right|^{2}$. 
TABLE I. Molecular vibrational modes and their chiral nonlinear amplitudes obtained from fitting SFG spectra with chiral polarization combinations.

\begin{tabular}{lcccc}
\hline \multicolumn{1}{c}{$\omega_{q} / 2 \pi c$} & $\Gamma_{q} / 2 \pi c$ & $A_{q} / 2 \pi c$ & $\left|\left(\alpha_{\text {chiral }}\right)_{q}\right|$ \\
\hline $\mathrm{CH}_{2}^{\text {sym }}$ & $2839 \mathrm{~cm}^{-1}$ & $11 \mathrm{~cm}^{-1}$ & $13 \times 10^{-12} \mathrm{~V}^{-1}$ & $1.3 \times 10^{-53} \frac{\mathrm{m}^{3} \mathrm{C}}{\mathrm{V}^{2}}$ \\
$\mathrm{CH}_{3}^{\text {sym }}$ & $2879 \mathrm{~cm}^{-1}$ & $12 \mathrm{~cm}^{-1}$ & $11 \times 10^{-12} \mathrm{~V}^{-1}$ & $1.0 \times 10^{-53} \frac{\mathrm{m}^{3} \mathrm{C}}{\mathrm{V}^{2}}$ \\
$\mathrm{CH}$ & $2905 \mathrm{~cm}^{-1}$ & $13 \mathrm{~cm}^{-1}$ & $-16 \times 10^{-12} \mathrm{~V}^{-1}$ & $1.3 \times 10^{-53} \frac{\mathrm{m}^{3} \mathrm{C}}{\mathrm{V}^{2}}$ \\
$\mathrm{CH}_{2}^{\text {asym }}$ & $2936 \mathrm{~cm}^{-1}$ & $11 \mathrm{~cm}^{-1}$ & $2.1 \times 10^{-12} \mathrm{~V}^{-1}$ & $0.2 \times 10^{-53} \frac{\mathrm{m}^{3} \mathrm{C}}{\mathrm{V}^{2}}$ \\
\hline \hline
\end{tabular}

We can also conclude from the absence of chiral spectra in reflection that the contribution from $\vec{\chi}_{S}^{(2)}$ to the chiral SFG signal observed in transmission is negligible (if any).

In conclusion, we observed, for the first time, optical activity in the SFG vibrational spectra of chiral liquids and thus proved the applicability of SFG as a vibrational spectroscopic tool to study chirality. The characteristic features appear as expected, but the chiral nonlinear polarizability element, which is electric-dipole allowed, is found to be about 3 orders of magnitude smaller than a typical achiral component. This can be understood from the fact that the observed effect requires a breakdown of the BornOppenheimer approximation to induce an antisymmetric component in the Raman polarizability associated with the vibrational resonances. That chirality is induced in inherently achiral atomic groups by perturbation results in further reduction. While the work reported here is focused on limonene, we have obtained similar results from other chiral liquids: carvone, menthylacetate, and 2-butanol.

This work was supported by the Director, Office of Energy Research, Office of Basic Energy Sciences, Material

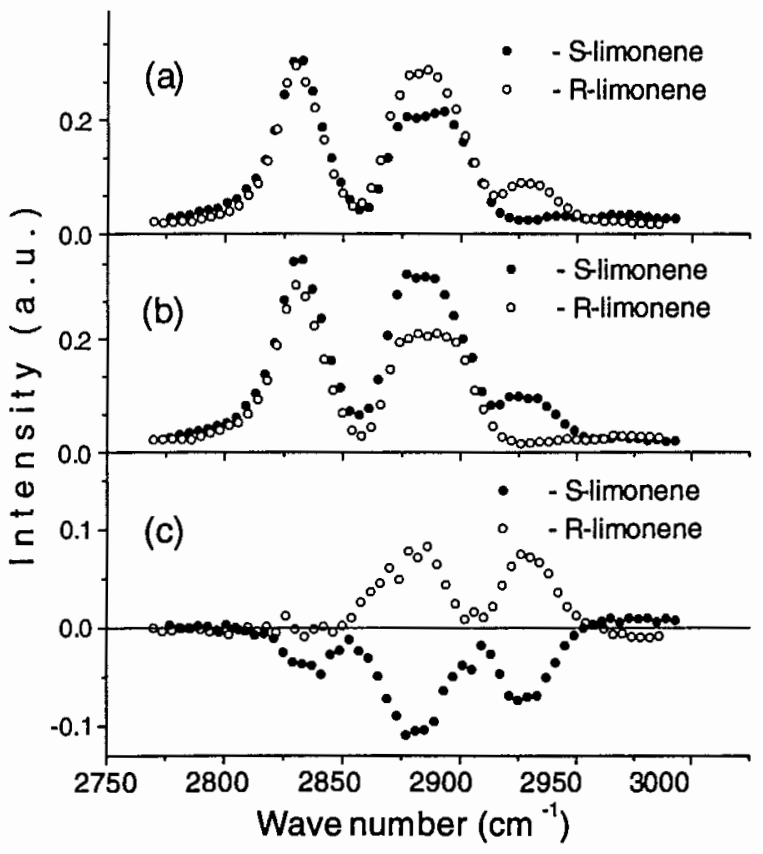

FIG. 4. Spectra of $S$ - and $R$-limonene in mixed polarization combinations: (a) $\left|\left(\chi_{\text {eff }}^{(2)}\right)_{+}\right|^{2}$, (b) $\left|\left(\chi_{\text {eff }}^{(2)}\right)_{-}\right|^{2}$, and (c) $\left|\left(\chi_{\text {eff }}^{(2)}\right)_{+}\right|^{2}-$ $\left|\left(\chi_{\text {eff }}^{(2)}\right)_{-}\right|^{2}$.
Science Division of the U.S. Department of Energy under Contract No. DE-AC03-76SF00098.

*Present address: Swiss Federal Institute for Materials Research, EMPA Dübendorf, Switzerland.

${ }^{\dagger}$ Present address: Department of Computer Science and Electrical Engineering, University of Maryland, College Park, Maryland.

₹To whom correspondence should be addressed. Email address: shenyr@physics.berkeley.edu

[1] See, for example, P. Ball, Designing the Molecular World (Princeton University Press, Princeton, NJ, 1996).

[2] See, for example, L. D. Barron, Molecular Light Scattering and Optical Activity (Cambridge University Press, Cambridge, UK, 1982).

[3] T. Petralli-Mallow et al., J. Phys. Chem. 97, 1383 (1993); J. D. Byers et al., Phys. Rev. B 49, 14643 (1994).

[4] J. J. Maki, M. Kauranen, and A. Persoons, Phys. Rev. B 51, 1425 (1995); M. Kauranen, T. Verbiest, and A. Persoons, J. Mod. Opt. 45, 403 (1998).

[5] Y. R. Shen, in Proceedings of the International School of Physics "Enrico Fermi," Course CXX, Frontiers in Laser Spectroscopy, edited by T.W. Hänsch and M. Inguscio (North-Holland, Amsterdam, 1994), p. 139.

[6] J. A. Giordmaine, Phys. Rev. 138, A1599 (1965).

[7] P. M. Rentzepis, J. A. Giordmaine, and K. W. Wecht, Phys. Rev. Lett. 16, 792 (1966).

[8] A.P. Shkurinov, A. V. Dubrovskii, and N. I. Koroteev [Phys. Rev. Lett. 70, 1085 (1993)] mentioned briefly that they had observed SFG from solution of chiral molecules, but it is not clear whether their SFG signal comes from chiral or achiral contribution. Recently, A. D. Buckingham and P. Fischer reported in the 219th ACS National Meeting [San Francisco, March, 2000, Paper No. 448 (to be published)] that they were not able to observe any chiral SFG signal in the solution used by Rentzepis et al. in Ref. [7].

[9] P. Guyot-Sionnest and Y. R. Shen, Phys. Rev. B 38, 7985 (1988).

[10] X. Wei et al., J. Phys. Chem. B 104, 3349 (2000).

[11] R. Superfine, J. Y. Huang, and Y. R. Shen, Chem. Phys. Lett. 172, 303 (1990).

[12] F. Liu, J. Phys. Chem 95, 7180 (1991).

[13] G. Herzberg, Molecular Spectra and Molecular Structure (Krieger, Malabar, FL, 1989-1991), 2nd ed.

[14] Estimated from the result by X. Wei et al. [Phys. Rev. Lett. 82, $4256(1999)]$. We assumed $\Gamma=10 \mathrm{~cm}^{-1}$. 